\title{
WEYL'S THEOREM FOR TENSOR PRODUCTS
}

\author{
YEONG-MOO SONG \\ Department of Mathematics Education, Sunchon National University, Sunchon 540-742, Korea \\ e-mail:ymsong@sunchon.ac.kr \\ and AN-HYUN KIM \\ Department of Mathematics, Changwon National University, Changwon 641-773, Korea \\ e-mail:ahkim@changwon.ac.kr
}

(Received 21 March, 2003; accepted 29 October, 2003)

\begin{abstract}
Suppose that $A$ and $B$ are 'isoloid' operators acting on a complex Banach space, that is, every isolated point of their spectra is an eigenvalue. In this note it is shown that if Weyl's theorem holds for both $A$ and $B$ then it holds for $A \otimes B$.
\end{abstract}

2000 Mathematics Subject Classification. 47A10, 47A53.

Throughout this note let $\mathcal{X}$ denote an infinite dimensional complex Banach space. Let $\mathcal{L}(\mathcal{X})$ denote the algebra of bounded linear operators on $\mathcal{X}$. If $T \in \mathcal{L}(\mathcal{X})$ write $N(T)$ and $R(T)$ for the null space and range of $T ; \sigma(T)$ for the spectrum of $T ; \pi_{0}(T)$ for the set of eigenvalues of $T$. Recall ([4], [5]) that $T \in \mathcal{L}(\mathcal{X})$ is called upper semi-Fredholm if it has closed range with finite-dimensional null space and lower semi-Fredholm if it has closed range with its range of finite co-dimension. If $T$ is either upper or lower semiFredholm, we call it semi-Fredholm and if $T$ is both upper and lower semi-Fredholm, we call it Fredholm. The index of a semi-Fredholm operator $T \in \mathcal{L}(\mathcal{X})$ is given by

$$
\operatorname{ind}(T)=\operatorname{dim} N(T)-\operatorname{dim} X / R(T) .
$$

An operator $T \in \mathcal{L}(\mathcal{X})$ is called $W e y l$ if it is Fredholm of index zero. The essential spectrum $\sigma_{e}(T)$ and the Weyl spectrum $\omega(T)$ of $T \in \mathcal{L}(\mathcal{X})$ are defined by

$$
\begin{aligned}
\sigma_{e}(T) & =\{\lambda \in \mathbb{C}: T-\lambda I \text { is not Fredholm }\} \\
\omega(T) & =\{\lambda \in \mathbb{C}: T-\lambda I \text { is not Weyl }\}:
\end{aligned}
$$

then (cf. [5])

$$
\sigma_{e}(T) \subseteq \omega(T) \subseteq \sigma_{e}(T) \cup \operatorname{acc} \sigma(T) \quad \text { and } \quad \omega(T) \subseteq \eta \sigma_{e}(T)
$$

where we write acc $K$ and $\eta K$ for the accumulation points and the polynomially-convex hull, respectively, of $K \subseteq \mathbb{C}$. We also write iso $K=K \backslash \operatorname{acc} K$ and

$$
\pi_{00}(T):=\left\{\lambda \in \text { iso } \sigma(T): 0<\operatorname{dim}(T-\lambda I)^{-1}(0)<\infty\right\}
$$

This paper was supported in part NON DIRECTED RESEARCH FUND from Suncheon National University. 
for the isolated eigenvalues of finite multiplicity. We say that Weyl's theorem holds for $T \in \mathcal{L}(\mathcal{X})$ if there is equality

$$
\sigma(T) \backslash \omega(T)=\pi_{00}(T) .
$$

H. Weyl [10] discovered that the equality $(0.1)$ holds for every hermitian operator. Weyl's theorem has been extended from hermitian operators to hyponormal operators and to Toeplitz operators by L. Coburn [3], to several classes of operators including seminormal operators by S. Berberian [1], [2], and to a few classes of Banach space operators [6], [7], [8]. In this note we examine Weyl's theorem for $A \otimes B$ when Weyl's theorem holds for $A$ and $B$.

Recall that an operator $T \in \mathcal{L}(\mathcal{X})$ is called an isoloid operator if iso $\sigma(T) \subseteq \pi_{0}(T)$, i.e., every isolated point of the spectrum is an eigenvalue (cf. [2], [8]).

Our main theorem now follows.

Theorem 1. Suppose $A, B \in \mathcal{L}(\mathcal{X})$ are isoloid. If Weyl's theorem holds for both $A$ and $B$ then it holds for $A \otimes B$.

Proof. We first show that

$$
\sigma(A \otimes B) \backslash \omega(A \otimes B) \subseteq \pi_{00}(A \otimes B) .
$$

To show this it suffices to show that $\sigma(A \otimes B) \backslash \omega(A \otimes B) \subseteq$ iso $\sigma(A \otimes B)$. Assume to the contrary that $\lambda \in \sigma(A \otimes B) \backslash \omega(A \otimes B)$ and $\lambda \in \operatorname{acc} \sigma(A \otimes B)$. Since $\lambda \in$ acc $(\sigma(A) \cdot \sigma(B))$, it follows that $\lambda \in[\operatorname{acc} \sigma(A) \cdot \sigma(B)] \cup[\sigma(A) \cdot \operatorname{acc} \sigma(B)]$ : indeed, more generally, if $H$ and $K$ are compact subsets of $\mathbb{C}$ then $\operatorname{acc}(H \cdot K) \subseteq[(\operatorname{acc} H) \cdot K] \cup[H$. (acc $K)]$. But since Weyl's theorem holds for $A$ and $B$, we have that acc $\sigma(A) \subseteq \omega(A)$ and $\operatorname{acc} \sigma(B) \subseteq \omega(B)$. Therefore

$$
\lambda \in \omega(A) \cdot \sigma(B) \cup \sigma(A) \cdot \omega(B)=\omega(A \otimes B),
$$

giving a contradiction. This proves (1). For the reverse inclusion we first observe

$$
\begin{gathered}
{[N(A) \otimes \mathcal{H}] \cup[\mathcal{H} \otimes N(B)] \subseteq N(A \otimes B) ;} \\
N(A-\mu I) \otimes N(B-\nu I) \subseteq N(A \otimes B-\mu \nu(I \otimes I)) \text { for each } \mu, v \in \mathbb{C}:
\end{gathered}
$$

indeed the inclusion (2) is evident and the inclusion (3) comes from the observation

$$
\begin{aligned}
{[A \otimes B-\mu \nu(I \otimes I)](x \otimes y) } & =[(A-\mu I) \otimes B+\mu I \otimes(B-v I)](x \otimes y) \\
& =(A-\mu I) x \otimes B y+\mu x \otimes(B-v I) y .
\end{aligned}
$$

Suppose $\lambda \in \pi_{00}(A \otimes B)$. We then proceed as follows.

Claim 1. $\lambda \neq 0$.

Claim 2. If $\lambda=\mu \nu$ with $\mu \in \sigma(A)$ and $\nu \in \sigma(B)$, then $\mu \in$ iso $\sigma(A)$ and $v \in$ iso $\sigma(B)$. Weyl.

Claim 3. If $\lambda=\mu \nu$ with $\mu \in \sigma(A)$ and $v \in \sigma(B)$ then $A-\mu I$ and $B-v I$ are both

For Claim 1, we assume to the contrary that $\lambda=0$. Thus $0 \in$ iso $\sigma(A \otimes B)$, and hence $0 \in$ iso $\sigma(A)$ or $0 \in$ iso $\sigma(B)$. But since $A$ and $B$ are isoloid it follows that $0 \in \pi_{0}(A)$ or $0 \in \pi_{0}(B)$. Therefore by (2) $N(A \otimes B)$ is infinite dimensional, which contradicts our assumption $0 \in \pi_{00}(A \otimes B)$. 
To prove Claim 2 we write $\lambda=\mu \nu$ with $\mu \in \sigma(A), v \in \sigma(B)$ and $\lambda \neq 0$. Assume to the contrary that $\mu \in \operatorname{acc} \sigma(A)$. Then we can find a sequence $\left\{\mu_{n}\right\}$ of distinct numbers in $\sigma(A)$ such that $\lim \mu_{n}=\mu$, so that $\lim \mu_{n} v=\lambda$, which shows that $\lambda \in \operatorname{acc} \sigma(A \otimes B)$, a contradiction; therefore $\mu \in$ iso $\sigma(A)$ and similarly $v \in$ iso $\sigma(B)$.

Towards Claim 3, note that $\mu \in \pi_{0}(A)$ and $v \in \pi_{0}(B)$ by Claim 2 because $A$ and $B$ are isoloid. We assume to the contrary that $A-\mu I$ is not Weyl. Thus $\mu \notin \pi_{00}(A)$ because $A$ obeys Weyl's theorem. So we have that $N(A-\mu I)$ is infinite dimensional. Also since $N(B-v I) \neq\{0\}$, it follows from (3) that $N(A \otimes B-\mu \nu(I \otimes I))$ is infinite dimensional, which contradicts our assumption $\lambda \in \pi_{00}(A \otimes B)$. This compltes the proof of Claim 3.

From Claims 1,2, and 3 we can conclude that if $\lambda \in \pi_{00}(A \otimes B)$ then $\lambda \notin \omega(A)$. $\sigma(B) \cup \sigma(A) \cdot \omega(B)$, and hence $\lambda \in \sigma(A \otimes B) \backslash \omega(A \otimes B)$. Therefore

$$
\pi_{00}(A \otimes B) \subseteq \sigma(A \otimes B) \backslash \omega(A \otimes B) .
$$

By (1) and (4) we can conclude that Weyl's theorem holds for $A \otimes B$.

EXAMPLE 2. (a) The "isoloid" condition is essential in Theorem 1. To see this let $T$ be an injective quasinilpotent operator on $\ell_{2}$ and define

$$
A=\left(\begin{array}{ccc}
1 & 0 & 0 \\
0 & 4 & 0 \\
0 & 0 & T+2
\end{array}\right): \mathbb{C} \oplus \mathbb{C} \oplus \ell_{2} \longrightarrow \mathbb{C} \oplus \mathbb{C} \oplus \ell_{2}
$$

Then

$$
\sigma(A)=\{1,2,4\}, \omega(A)=\{2\}, \text { and } \pi_{00}(A)=\{1,4\}
$$

so Weyl's theorem holds for $A$, while

$$
\begin{aligned}
\sigma(A \otimes A) & =\sigma(A) \cdot \sigma(A)=\{1,2,4,8,16\} ; \\
\omega(A \otimes A) & =\sigma(A) \cdot \omega(A)=\{2,4,8\} ; \\
\pi_{00}(A \otimes A) & =\{1,4,16\} ;
\end{aligned}
$$

so Weyl's theorem fails for $A \otimes A$. Note that $A$ is not isoloid.

(b) On the other hand, the condition "Weyl's theorem holds for both $A$ and $B$ " is essential in Theorem 1. If Weyl's theorem does not hold for either $A$ or $B$, then Theorem 1 may fail. To see this, consider the operators on $\ell_{2} \oplus \ell_{2}$ defined by

$$
A=U \oplus U^{*} \quad \text { and } \quad B=\left(I-U U^{*}\right) \oplus 0_{\infty},
$$

where $U$ is the unilateral shift on $\ell_{2}$. Let $\mathbb{D}$ and $\mathbb{T}$ denote the closed unit disk and the unit circle, respectively. Then we have that (i) $A$ and $B$ are both isoloid; (ii) $\sigma(A)=\mathbb{D}$ and $\omega(A)=\mathbb{T}$, and hence Weyl's theorem fails for $A$; (iii) $\sigma(B)=\{0,1\}, \omega(B)=\{0\}$ and $\pi_{00}(B)=\{1\}$, and hence Weyl's theorem holds for $B$; (iv) $\sigma(A \otimes B)=\mathbb{D}$ and $\omega(A \otimes$ $B)=\mathbb{} \cup\{0\}$, and hence Weyl's theorem fails for $A \otimes B$.

(c) The converse of Theorem 1 may not be true in general. Indeed if $A=U \oplus U^{*}$ as in (b) then $\sigma(A \otimes 1)=\mathbb{D}, \omega(A \otimes 1)=\mathbb{D}$ and $\pi_{00}(A \otimes 1)=\emptyset$, which implies that Weyl's theorem holds for $A \otimes 1$ although $A$ does not satisfy Weyl's theorem. 
If $\mathcal{H}$ is a complex Hilbert space and $T \in \mathcal{L}(\mathcal{H})$, write $W(T)$ for the numerical range of $T$. It is also familiar that $W(T)$ is convex and conv $\sigma(T) \subseteq \mathrm{cl} W(T)$. An operator $T$ is called convexoid if conv $\sigma(T)=\mathrm{cl} W(T)$. Also $T$ is called restriction-convexoid if the restriction of $T$ to every invariant subspace is convexoid and is called reductionconvexoid if every direct summand of $T$ is convexoid. It is known [2] that hyponormal $\Rightarrow$ restriction-convexoid $\Rightarrow$ isoloid.

COROLlary 3. If $\mathcal{H}$ is a complex Hilbert space and $A, B \in \mathcal{L}(\mathcal{H})$ are restrictionconvexoid then Weyl's theorem holds for $A \otimes B$.

Proof. By an argument of Prasanna [9, Theorem 2.1], Weyl's theorem holds for restriction-convexoid operators. Thus the result immediately follows from Theorem 1.

Weyl's theorem may fail for reduction-convexoid operators. For example if $A=$ $U \oplus U^{*}$, where $U$ is the unilateral shift on $\ell_{2}$, then $A$ is reduction-convexoid because $U$ and $U^{*}$ are both convexoid and have no nontrivial reducing subspaces, while Weyl's theorem fails for $A$. Note that $A$ is not restriction-convexoid.

ACKNOWLEDGEMENT. The authors are grateful to the referee for helpful comments concerning this paper.

\section{REFERENCES}

1. S. K. Berberian, An extension of Weyl's theorem to a class of not necessarily normal operators, Michigan Math. J. 16 (1969), 273-279.

2. S. K. Berberian, The Weyl spectrum of an operator, Indiana Univ. Math. J. 20 (1970), $529-544$ $285-288$

3. L. A. Coburn, Weyl's theorem for nonnormal operators, Michigan Math. J. 13 (1966),

4. R. E. Harte, Fredholm, Weyl and Browder theory, Proc. Royal Irish Acad. Sect. A 85 (1985), 151-176.

5. R. E. Harte, Invertibility and singularity for bounded linear operators (Dekker, New York, 1988).

6. V. I. Istrătescu, On Weyl's spectrum of an operator. I, Rev. Roum. Math. Pures Appl. 17 (1972), 1049-1059.

7. W. Y. Lee and H. Y. Lee, On Weyl's theorem, Math. Japan 39 (1994), 545-548.

8. K. K. Oberai, On the Weyl spectrum, Illinois J. Math. 18 (1974), 208-212. $59-63$.

9. S. Prasanna, Weyl's theorem and thin spectra, Indian Acad. Sci. (Math. Sci.) 91 (1982),

10. H. Weyl, Über beschränkte quadratische Formen, deren Differenz, vollsteig ist, Rend. Circ. Mat. Palermo 27 (1909), 373-392. 